\title{
GLOBALLY OPTIMAL DISPLACEMENT FIELDS USING LOCAL TENSOR METRIC
}

\author{
Gustaf Johansson $^{\star \dagger} \quad$ Daniel Forsberg ${ }^{\star \dagger \ddagger} \quad$ Hans Knutsson ${ }^{\star \dagger}$ \\ * Department of Biomedical Engineering, Linköping University, Sweden \\ ${ }^{\dagger}$ Center for Medical Image Science and Visualization (CMIV), Linköping University, Sweden \\ $\ddagger$ Sectra Imtec, Linköping, Sweden
}

\begin{abstract}
In this paper, we propose a novel algorithm for regularizing displacement fields in image registration. The method uses the local structure tensor and gradients of the displacement field to impose a local metric, which is then used optimizing a global cost function. The method allows for linear operators, such as tensors and differential operators modeling the underlying physical anatomy of the human body in medical images. The algorithm is tested using output from the Morphon image registration algorithm on MRI data as well as synthetic test data and the result is compared to the initial displacement field. The results clearly demonstrate the power of the method and the unique features brought forth through the global optimization approach.
\end{abstract}

Index Terms - Image Processing, Image Registration, Regularization, Optimization, Tensor

\section{INTRODUCTION}

Image registration is a well-known concept, frequently applied in a number of different areas, for instance geophysics, robotics and medicine. Its application range within medicine is vast and it has been proven to be a useful tool, both in research and in clinical practice. The basic idea of image registration is to find a displacement field $\mathbf{d}$ that geometrically transforms a template image $I_{T}$ in order to align it with a reference image $I_{R}$. A general classification of different image registration algorithms is to classify them as either parametric or non-parametric [1]. Parametric methods refers to methods, where a parametrization has been performed to reduce the number of degrees of freedom in the transformation space, this includes rigid, affine or spline-based registration. Nonparametric methods on the other hand independently estimate a displacement vector for each data point.

Regularization of the displacement field is an important aspect of image registration. It is needed to smooth the displacement field in order to ensure a plausible transformation/deformation, especially in the case of non-parametric registration. The regularizer is not only used to smooth the displacement field but can also be used to model the displacement field. A common approach is to model the displacement field according to various physical processes. This is for instance the case when applying fluid or elastic regularization, where fluid regularization can be explained as painting the image on honey [2] while elastic regularization refers to when the image is painted on a piece of rubber [3].

Another approach in regularization is to allow information in the image data itself to drive the registration (datadriven regularization). Examples of this include normalized convolution with the local structure as certainty [4], adaptive isotropic Gaussian convolution [5], direction dependent regularization [6], adaptive regularization based upon local noise level and local structural content [7] or adaptive anisotropic regularization based upon local structure [8]. Most of the described data-driven regularizers rely on the fact that structural content in the image should control the regularization, i.e. high certainty of local structural content ensures high certainty of the local displacement field.

The method presented in this paper is a data-driven regularizer. It allows for defining cost-functions that are representations of a range of models in physics and engineering that can be expressed in terms of linear operations such as differential equations and tensors. This allows the proposed method to be used in a number of different scenarios. The main objective of the cost function defined in this work is to achieve a displacement field that is as smooth as possible without allowing the field to alter the shape of salient local structures.

\section{METHOD}

\subsection{Global regularization}

Regularization has the objective of smoothing the displacement field in a way that it does not significantly increase the matching error. The physical properties of the imaged medical volume imposes certain requirements on the displacement fields, e.g. motion of rigid objects and sliding along organ surfaces.

The behavior of the global regularization is defined through the cost function. The first term in the cost function used in the present work contains a gradient forcing the spatial partial derivatives of the updated field to be small. The objective of the second term is to only allow changes to 
the field in areas with little or insignificant structural content. The local structure tensor [9] of the reference image is used to decide in which directions the update field is allowed to be smoothed. Finally, a parameter $\alpha$ is introduced. This parameter is used to decide the relative importance of displacement field smoothing versus conservation of structures. The optimization problem solved in this paper, is stated as:

$$
\mathbf{v}_{0}=\underset{\mathbf{v}}{\operatorname{argmin}} \underbrace{\left[(1-\alpha)\|\nabla(\mathbf{v}+\mathbf{d})\|^{2}+\alpha\|\mathbf{T} \mathbf{v}\|^{2}\right]}_{f}
$$

$$
\begin{array}{llcl}
\mathbf{v}_{0} & \text { Optimal update } & \alpha & \text { Rel. weight parameter } \\
\mathbf{d} & \text { Initial displacement } & \nabla & \text { Gradient } \\
\mathbf{v} & \text { Displacement update } & \mathbf{T} & \text { Structure tensor }
\end{array}
$$

!The first term consists of a squared Frobenius norm of the gradient of the displacement field. The gradient is a linear operation and can be described by a matrix multiplication corresponding to spatial derivatives. The second term consists of the structure tensor of the images corresponding to the initial displacement field operating on the displacement initial field. The tensor acts as a linear operator and can also be represented by a matrix. The problem stated can now be rewritten using a matrix representation. To this end we will use a vectorization of the displacement field:

$$
\mathbf{d}=\left(\begin{array}{ccc}
\mathbf{d}_{1}^{T} & \ldots & \mathbf{d}_{D}^{T}
\end{array}\right)^{T}
$$

Denoting the matrices of the gradient and the tensor as $\mathbf{M}_{1}$ and $\mathbf{M}_{2}$ respectively and realizing that the Frobenius norm in practice is a scalar product, there is a matrix representation of objective function, $f$.

$$
f=(1-\alpha)(\mathbf{d}+\mathbf{v})^{T} \mathbf{M}_{1}^{T} \mathbf{M}_{1}(\mathbf{d}+\mathbf{v})+\alpha \mathbf{v}^{T} \mathbf{M}_{2}^{T} \mathbf{M}_{2} \mathbf{v}
$$

$f$ contains nothing more complicated than a sum of quadratic forms, linear and constant terms. Differentiating $f$ with respect to $\mathbf{v}$ and setting equal to 0 results in a linear equation system. Solving yields the solution:

$$
\mathbf{v}_{0}=\underbrace{-\left[\mathbf{M}_{1}^{T} \mathbf{M}_{1}+\frac{\alpha}{1-\alpha} \mathbf{M}_{2}^{T} \mathbf{M}_{2}\right]^{-1} \mathbf{M}_{1}^{T} \mathbf{M}_{1}}_{\mathbf{M}} \mathbf{d}
$$

This can be viewed as calculating a matrix, $\mathbf{M}$, to operate on the displacement field, $\mathbf{d}$. $\mathbf{M}$ is a linear operator, which yields the update field, $\mathbf{v}$, from the displacement field $\mathbf{d}$. Its rows can then actually be viewed as a vectorization of a linear spatially variant filter that is optimized with respect to the data and the cost function. A couple of such adaptive filters are presented in figure 2.

Understanding that a spatial differentiation $\frac{\partial\left(\cdot{ }^{\prime}\right)}{\partial\left(\cdot{ }_{j}\right)}$ will be represented with a square matrix of same side as the number of elements in the dimension, denote these matrices $\mathbf{G}_{i, j}$. The special transpose operator ${ }^{\top}$ denotes transpose of the outer structure and does not transpose the individual block matrices. In terms of a block matrix, the $\mathbf{M}_{1}$ matrix becomes a $D^{2} \times D$ matrix of blocks if $D$ is the number of dimensions:

$$
\mathbf{M}_{1}=\left(\begin{array}{llll}
\mathbf{G}_{\cdot, 1} & \mathbf{G}_{\cdot, 2} & \ldots & \mathbf{G}_{\cdot, D}
\end{array}\right)^{\top}
$$

The intermediate level block-matrices are:

$$
\mathbf{G}_{., k}=\left(\begin{array}{cccc}
\mathbf{G}_{1, k} & \mathbf{0} & \ldots & \mathbf{0} \\
\mathbf{0} & \mathbf{G}_{2, k} & \ldots & \mathbf{0} \\
\vdots & \vdots & \ddots & \vdots \\
\mathbf{0} & \mathbf{0} & \ldots & \mathbf{G}_{D, k}
\end{array}\right)
$$

Let $\mathbf{T}_{i, j}$ be matrix representation of component $t_{i j}$ in the structure tensor, then the representation could be written

$$
\mathbf{M}_{2}=\left(\begin{array}{cccc}
\mathbf{T}_{1,1} & \mathbf{T}_{1,2} & \ldots & \mathbf{T}_{1, D} \\
\mathbf{T}_{2,1} & \mathbf{T}_{2,2} & \ldots & \mathbf{T}_{2, D} \\
\vdots & \vdots & \ddots & \vdots \\
\mathbf{T}_{D, 1} & \mathbf{T}_{D, 2} & \ldots & \mathbf{T}_{D, D}
\end{array}\right)
$$

The total equation system size is polynomial in the number of data elements, $N$, and exponential in number of dimensions. The number of nonzero entries are, however, linear in data size allowing a computationally efficient solution, i.e we get:

$$
\mathcal{O}\left(N^{2 D}\right) \text { - matrix elements, } \mathcal{O}(N D) \text { - nonzero elements. }
$$

To illustrate an example, in the special case of 2D used in this paper, the two matrices become:

$$
\begin{gathered}
\mathbf{M}_{1}=\left(\begin{array}{cccc}
\mathbf{G}_{1,1} & \mathbf{0} & \mathbf{G}_{1,2} & \mathbf{0} \\
\mathbf{0} & \mathbf{G}_{2,1} & \mathbf{0} & \mathbf{G}_{2,2}
\end{array}\right)^{\top} \\
\mathbf{M}_{2}=\left(\begin{array}{cc}
\mathbf{T}_{1,1} & \mathbf{T}_{1,2} \\
\mathbf{T}_{2,1} & \mathbf{T}_{2,2}
\end{array}\right)
\end{gathered}
$$

Further, finding the matrix operation is not necessary to find the matrix inverse involved, one merely needs to perform a matrix division where the right hand side turns out to be a vector, greatly reducing the computational load. In terms of speed, an algorithm implemented in Matlab easily executes in roughly one second for the example data used in this paper.

\subsection{Initial displacement field - The Morphon}

In this paper we have combined the proposed regularizer with an image registration algorithm, known as the Morphon, to demonstrate the usefulness of the regularizer. The Morphon is a phase-based algorithm where a template image $I_{T}(\mathbf{x})$ is iteratively deformed, until it fits a reference image $I_{R}(\mathbf{x})$. This process is performed over multiple scales starting on coarse scales to register large global displacements and moving on to finer scales to register smaller local deformations. The algorithm itself consists of the following three steps: local displacement estimation, displacement field accumulation, deformation. For a more detailed review of the different steps the user is referred to [10]. 
In each iteration the local displacement estimation is based upon a quadrature phase-difference estimation. The deformed template and reference images are filtered with a set of quadrature filters $f_{k}$ with $K$ different orientations $\hat{\mathbf{n}}_{k}$. The filter outputs,

$$
q_{D_{k}}=I_{D} * f_{k} \quad \text { and } \quad q_{R_{k}}=I_{R} * f_{k},
$$

describing how edge- or line-like the local neighborhood is, are used to compute local phase-difference based displacement estimates $d_{k}$ for each filter direction, $k$.

The displacement estimates and the coupled certainties $c_{k}$ are then used to compute an incremental displacement field $\mathbf{d}_{i}$. This is done by finding the solution to a least square problem defined as:

$$
\mathbf{d}_{i}=\underset{\mathbf{d}}{\operatorname{argmin}} \sum_{k=1}^{K}\left[c_{k} \mathbf{T}_{R}\left(d_{k} \hat{\mathbf{n}}_{k}-\mathbf{d}\right)\right]^{2}
$$

where $\mathbf{d}_{i}$ is the estimated incremental displacement and $\mathbf{T}_{R}$ is the local structure tensor of $I_{R}$.

The incremental displacement field and certainty are then added to the accumulated displacement field $\mathbf{d}_{a}$ and certainty $c_{a}$ according to:

$$
\begin{aligned}
\mathbf{d}_{a} & =\frac{c_{a} \mathbf{d}_{a}+c_{i}\left(\mathbf{d}_{a}+\mathbf{d}_{i}\right)}{c_{a}+c_{i}} \\
c_{a} & =\frac{c_{a}^{2}+c_{i}\left(c_{a}+c_{i}\right)}{c_{a}+c_{i}}
\end{aligned}
$$

To obtain a smoothly varying displacement field the accumulated displacement field is locally regularized in each iteration. The regularization is achieved by normalized averaging [11] using a Gaussian low pass kernel $g$ as filter and $c_{a}$ as certainty.

The last iteration step is to use the accumulated displacement field to deform the template image.

$$
I_{D}(\mathbf{x})=I_{T}\left(\mathbf{x}+\mathbf{d}_{a}(\mathbf{x})\right)
$$

It's the final displacement field, $\mathbf{d}_{a}$, of these iterations that provides the input to the presented global regularization approach.

\section{RESULTS}

\subsection{Quantitative measures}

Two values that are used as a measure of the smoothness of the displacement field is the laplacian root-mean-square (L-RMS), and the gradient root-mean-square (G-RMS). Two similarity measures between images that are commonly used are normalized cross-correlation (NCC) and mutual information (MI). In table 1 these measures are evaluated for a set of different $\alpha$.

\begin{tabular}{ccccc}
\hline$\alpha$ & NCC & MI & G-RMS & L-RMS \\
\hline 1.00 & 0.967 & 1.639 & 0.333 & 0.123 \\
0.99 & 0.967 & 1.633 & 0.282 & 0.087 \\
0.95 & 0.967 & 1.622 & 0.239 & 0.065 \\
0.90 & 0.966 & 1.617 & 0.217 & 0.055 \\
0.80 & 0.966 & 1.608 & 0.194 & 0.045 \\
0.50 & 0.964 & 1.592 & 0.155 & 0.030 \\
0.10 & 0.954 & 1.538 & 0.093 & 0.013 \\
\hline
\end{tabular}

Table 1. Similarity measures for various values of $\alpha$. The images used are the Brain MRI images in figure 3. $\alpha=1.00$ is the same as no smoothing.
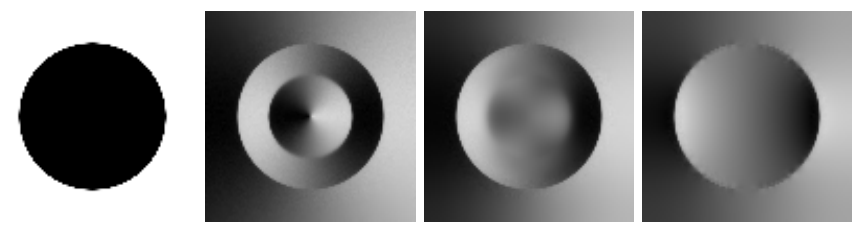

Fig. 1. Left: Object being registered. Three rightmost images: The $\mathrm{x}$-component of the regularized displacement field, using three different values of $\alpha$ respectively. Note that the field is gracefully preserved around the circle structure, whereas the parts of the field where the image lacks structure are smoothed considerably.

\subsection{Qualitative measures}

In this section some images are presented to demonstrate the power of the global method when it comes to regularize nonlocal points in the fields. The effects on one synthetic image with corresponding field in figure 1 . There is also an example of the Brain images used in the quantitative study: images in figure 3 and displacement fields in figure 4. In Table 1 various metrics are presented before as well as after the regularization of the registration of a 2D MRI slice.
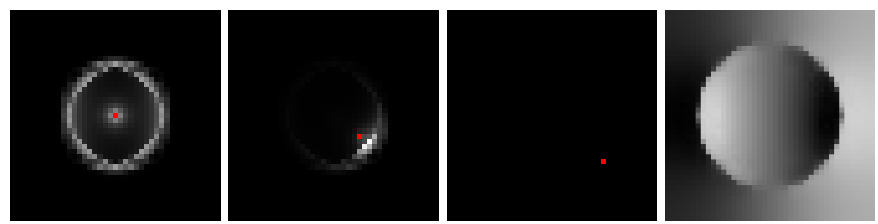

Fig. 2. Color coded optimized filter for the $x$-component at three different positions. Filter coefficients are in gray scale, position of application is in red. Note the point on the edge where no smoothing is allowed. Right: Resulting regularization of $\mathrm{x}$-component. 

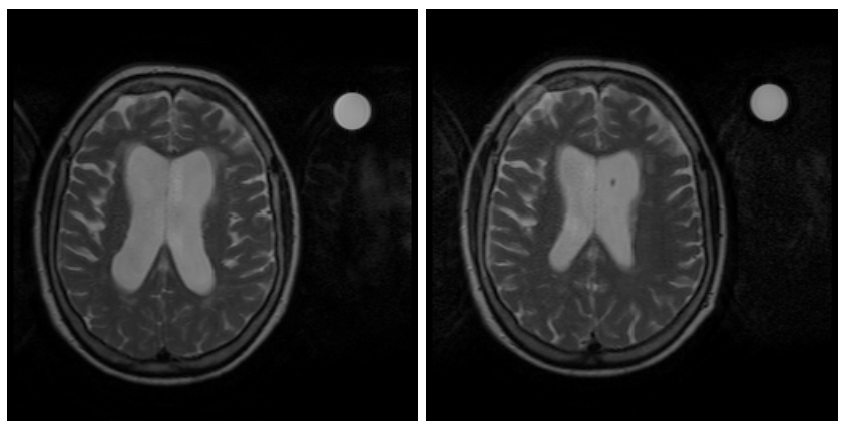

Fig. 3. Left: MRI slice of a brain before surgery Right: Same slice after surgery.
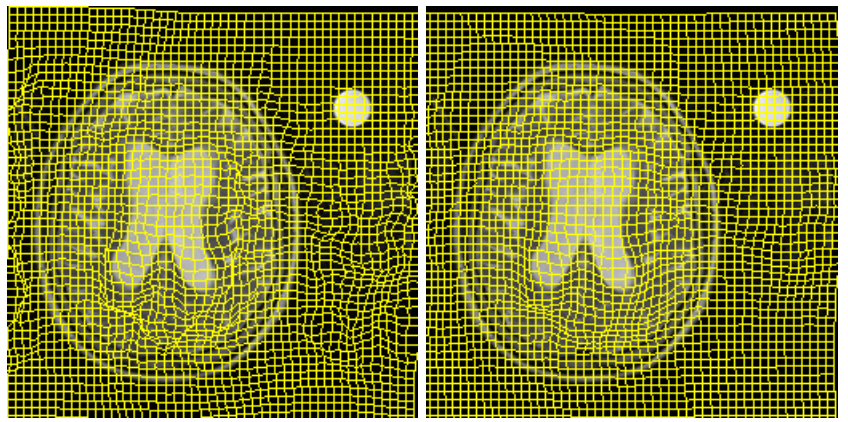

Fig. 4. Left: Before regularization. Right: After regularization.

\section{DISCUSSION}

Qualitatively, the Brain MRI and the synthetic example images illustrate that the claimed quality of regularizing nonstructural parts of the field while preserving structures is actually achieved. Quantitatively, the results in table 1 show that the method presented makes it possible to make the displacement fields considerably smoother without significantly decreasing neither the NCC nor MI measures. Further, the optimization framework presented is very general and is possible to extend to any linear transformation including for instance spatially varying linear filters as well as linear operations such as tensor products and higher order differential operators - yielding a large range of opportunities for adding new terms to the cost function and, in this way, designing more advanced, sophisticated and specialized algorithms.

\section{ACKNOWLEDGMENTS}

This work was supported by the Swedish Research Council (grant 2007-4786) and the Linneaus center CADICS. Also thanks to colleague Anders Eklund for advice and proofreading.

\section{REFERENCES}

[1] J. Modersizki, Numerical Methods for Image Registration, Oxford University Press, 2004.

[2] G.E. Christensen, R.D. Rabbitt, and M.I. Miller, "Deformable templates using large deformation kinematics," Image Processing, IEEE Transactions on, vol. 5, no. 10, pp. $1435-1447$, oct 1996.

[3] C. Broit, Optimal registration of deformed images, Ph.D. thesis, 1981.

[4] E. Suarez, C-F. Westin, E. Rovaris, and J. RuizAlzola, "Nonrigid registration using regularized matching weighted by local structure," in Medical Image Computing and Computer-Assisted Intervention MICCAI 2002, vol. 2489 of Lecture Notes in Computer Science, pp. 581-589. 2002.

[5] R. Stefanescu, X. Pennec, and N. Ayache, "Grid powered nonlinear image registration with locally adaptive regularization," Medical Image Analysis, vol. 8, no. 3, pp. 325-342, 2004.

[6] A. Schmidt-Richberg, J. Ehrhardt, R. Werner, and H. Handels, "Slipping objects in image registration: Improved motion field estimation with direction-dependent regularization," in Medical Image Computing and Computer-Assisted Intervention MICCAI 2009, vol. 5761 of Lecture Notes in Computer Science, pp. 755762. 2009.

[7] L. Tang, G. Hamarneh, and R. Abugharbieh, "Reliability-driven, spatially-adaptive regularization for deformable registration," in Biomedical Image Registration, vol. 6204 of Lecture Notes in Computer Science, pp. 173-185. 2010.

[8] D. Forsberg, M. Andersson, and H. Knutsson, "Adaptive anisotropic regularization of deformation fields for non-rigid registration using the morphon framework," in ICASSP, Dallas, USA, March 2010.

[9] H. Knutsson, "Representing local structure using tensors," in The 6th Scandinavian Conference on Image Analysis, June 1989, pp. 244-251.

[10] H. Knutsson and M. Andersson, "Morphons: Segmentation using elastic canvas and paint on priors," in IEEE International Conference on Image Processing (ICIP'05), Genova, Italy, September 2005.

[11] H. Knutsson and C-F. Westin, "Normalized and differential convolution: Methods for interpolation and filtering of incomplete and uncertain data," in Proceedings of IEEE Computer Society Conference on Computer Vision and Pattern Recognition, New York City, USA, June 1993, IEEE, pp. 515-523. 